\title{
Propedéutica y práctica de la investigación según los programas de Metodología en carreras de Sociología en Argentina y otros países latinos
}

Propedeutics and practice of research in Methodology programs of undergraduate Sociology degrees in Argentina and other Latin American countries

Propedèutica i pràctica de la investigació segons els programes de Metodologia en carreres de Sociologia a Argentina i altres països Ilatins

\author{
Gonzalo Seid $^{*}$, Federico Luis Abiuso ${ }^{\circledR}$ \\ Instituto de Investigaciones Gino Germani, Universidad de Buenos Aires, Argentina \\ *Autor para correspondencia: gonzaloseid@gmail.com (Gonzalo Seid)
}

Recibido: 06/04/2020 | Aceptado: 14/10/2020 | Publicado: 22/12/2020

\section{Cómo citar: Seid, G., y Abiuso, F.L (2020). Propedéutica y práctica de la investigación según los programas de Metodología en carreras de Sociología en Argentina y otros países latinos. Research in Education and Learning Innovation Archives, 25,1-16.}

10.7203/realia.25.17001

Copyright: El/La Autor/a. Open Access: Este es un artículo de acceso abierto distribuido bajo los términos de la licencia Creative Commons

Attribution-NoDerivatives 4.0 International licence (CC BY-ND 4.0)

\footnotetext{
Financiación: "Evaluando recursos pedagógicos para la articulación de teoría y metodología de la investigación". Proyecto de Reconocimiento Institucional de la Facultad de Ciencias Sociales, Universidad de Buenos Aires, Programación 2018-2020
}

RESUMEN: Los programas de materias de Metodología de la Investigación en carreras universitarias de grado en Sociología, principalmente de Argentina (2018), constituyen el objeto de análisis del presente artículo. Partiendo de interrogantes en torno a los modos en que se enseña a investigar, se escogió analizar programas en tanto documentos que cristalizan y brindan indicios de prácticas y discursos formativos. Si bien los programas distan de las prácticas en las aulas, ofrecen información significativa y permiten ampliar la muestra respecto a la observación in situ. Los resultados del relevamiento se organizaron en dos ejes: las variantes en las propuestas de enseñanza y las maneras de entender las prácticas de investigación. En el corpus analizado se encontró que existe un amplio consenso en el plano discursivo en torno a la idea "Se aprende a investigar investigando". Sin embargo, el grado y las modalidades de aplicación práctica de los conocimientos conceptuales parecen diferir según los temas que se requiera enseñar. Las técnicas y operaciones más concretas son las que aparecen más ligadas a la puesta en práctica, pero no siempre las técnicas se enseñan enmarcándolas en una investigación completa. En el artículo se señala la importancia de las distintas maneras de entender qué es la investigación y qué es una práctica. Se concluye que, al existir mandatos contradictorios para la enseñanza de metodología de la investigación social, los diversos abordajes docentes reflejan esas tensiones y resuelven de distintas formas los desafíos.

PALABRAS CLAVE: investigación; enseñanza universitaria; metodología; sociología; propedéutica

ABSTRACT: In this article we discuss the teaching programs of Research Methodology subjects imparted in undergraduate Sociology degrees, mainly in Argentina (2018). Starting with questions about how research is taught, we analyzed programs as documents that develop and deliver information on formative practices and discourses. While these programs are by no means classroom practices, they provide significant information and help to increase the sample compared to observation in situ. Our results are divided into two axes: variations in teaching proposals and ways of understanding research practices. The corpus we analyzed reflects a broad consensus on the discursive level around the idea "You learn to investigate by investigating". However, the extent and modes of the practical application of theoretical knowledge appear to depend on which topics need teaching. The most concrete techniques and operations are those that appear most related to their practical 
implementation. However, these techniques and operations are not always taught by framing them within a comprehensive investigative study. We highlight the importance of taking into account the conceptions about what is research and what is practice. We conclude that, since there are contradictory opinions on the teaching of social research methodology, the range of teaching approaches reflects these tensions and meets the various challenges in different ways.

KEYWORDS: research; Higher education; methodology; Sociology; propedeutics

RESUM: Els programes de la matèria Metodologia de la Investigació en carreres universitàries de grau en Sociologia, principalment d'Argentina (2018), constitueixen l'objecte d'anàlisi d'aquest article. Partint d'interrogants sobre com s'ensenya a investigar, hem triat analitzar els programes en documents que plasmen i proporcionen indicis de pràctiques i discursos formatius. Si bé els programes són lluny de la pràctica a l'aula, ofereixen informació significativa i permeten ampliar la mostra en comparació amb l'observació in situ. Els resultats de la investigació es van organitzar en dos eixos: les variants en les propostes d'ensenyament i les maneres d'entendre les pràctiques d'investigació. En el corpus analitzat es va trobar que hi ha un ample consens pel que fa al nivell discursiu sobre la idea "S'aprèn a investigar investigant". Tanmateix, el grau i les modalitats de l'aplicació pràctica dels coneixements teòrics semblen diferir segons que siguen els temes que han de ser ensenyats. Les tècniques i activitats més concretes són les que apareixen més lligades a l'aplicació pràctica, però no sempre les tècniques s'ensenyen emmarcant-les en una investigació completa. Hem de remarcar que és molt important tenir en compte el concepte que es té de la investigació i del significat que en cada cas implica la pràctica. Es conclou que, per tal com hi ha visions contradictòries per a l'ensenyament de la metodologia en investigació social, els diferents enfocaments docents reflecteixen aquestes tensions i resolen de manera diferent els desafiaments.

PARAULES CLAU: investigació; ensenyament universitari; metodologia; sociologia; propedèutica

\section{Notas de aplicación práctica}

\section{Qué se sabe sobre el tema}

- Se espera que la enseñanza universitaria de Metodología de la Investigación Social esté orientada a la práctica de la investigación, pero el hecho de que estos contenidos constituyan materias en los planes de estudio los separa de la teoría social y lleva a adaptarlos a las posibilidades del aula.

\section{Qué aporta este trabajo}

- Se analizan programas de materias de Metodología de la Investigación de carreras de Sociología de varias universidades, evidenciando la variabilidad en las maneras de entender las prácticas.

\section{Implicaciones para la práctica y/o política}

- Parece recomendable que las carreras incluyan materias metodológicas con distintas modalidades de enseñanza, o bien que puedan identificar y compensar las limitaciones del modelo de enseñanza elegido.

\section{INTRODUCCIÓN}

La enseñanza de la investigación carga con responsabilidades contradictorias ${ }^{1}$. Una de ellas remite a desarrollar un espíritu creativo y promover la autonomía intelectual. Tener presente que no hay recetas, que todos somos capaces de producir conocimiento, que saber más no siempre implica investigar mejor. En definitiva, que enseñar a investigar es hacer como el profesor facotot que rescata (Rancière, 2007) enseñar lo que no se sabe. El otro mandato, siguiendo a (Bourdieu y Passeron, 2004, 110) apunta a "que se amplíe el dominio de lo que puede ser racional y técnicamente adquirido a través de un aprendizaje metódico a expensas de lo que es abandonado irreductiblemente

\footnotetext{
${ }^{1}$ Agradecemos a los evaluadores anónimos de la revista por sus comentarios para mejorar este trabajo.
} 
al azar de los talentos individuales, es decir, de hecho, a la lógica de los privilegios sociales". Esto supone evitar dar por sentados determinados saberes y competencias, lo que produciría "lagunas inadmisibles", especialmente en lo que respecta a "modos de pensar o de saber-hacer fundamentales que, al considerarse como enseñados por todo el mundo, acaban por no ser enseñados por nadie" (Bourdieu y Gros,1998, 132).

En el presente artículo $^{2}$ se analizan algunos programas de materias sobre metodología de la investigación de carreras de Sociología de distintas cátedras y universidades, con el fin de tipificar las propuestas de enseñanza-aprendizaje que allí se presentan. Se relevaron programas de materias metodológicas del año 2018 de las carreras de Sociología de universidades públicas argentinas -al menos un programa por carrera. $\mathrm{Al}$ avanzar el análisis se decidió ampliar la muestra incluyendo algunos programas de otros países (México, Italia y España). Como presentación de los resultados, el artículo se organiza del siguiente modo. En primer lugar, se consignan antecedentes en la temática de enseñanza de la investigación. Luego, se detallan las decisiones metodológicas de la presente indagación, referidas a la selección de casos, la codificación y el análisis de los programas relevados. En tercer lugar, se presentan los resultados: se describen las variantes en las propuestas de enseñanza y se distinguen maneras de entender y poner en práctica la investigación que subyacen los programas.

\section{ANTECEDENTES}

En numerosas ocasiones se ha afirmado que a investigar se aprende investigando (Bourdieu, Chamboredon, y Passeron, 2008; Rizo-García, 2006; Wainerman y Sautu, 2011). Esto implica que el aprendiz participe de un equipo de trabajo donde pueda ser guiado por profesionales formados que compartan su experiencia y le enseñen algunos "trucos" para resolver obstáculos concretos de cada investigación (Becker, 2009). Las carreras universitarias de grado en Sociología no suelen estar organizadas según este modelo, pero sí existen preocupaciones y esfuerzos en esa dirección. En general, los diseños curriculares de las carreras de Sociología en América Latina ubican a la metodología de la investigación como una materia separada de otros dominios de conocimiento, como modo de resolver el problema pedagógico de la enseñanza de investigación a un estudiantado masivo. La materia Metodología de la Investigación "suscita múltiples voces a favor y en contra de, por un lado, el propio dictado de la misma y, luego, de sus contenidos". Algunas críticas se fundamentan en "cierta imposibilidad aprender (aprehender) a investigar sin investigar, limitado al espacio de un aula, junto con la necesidad de incorporar saberes teóricos y prácticos” (Gómez-Rojas y Malegarie, 2007, 2).

Un antecedente relevante en Argentina es el trabajo coordinado por Cohen y Piovani (2008) donde se problematiza la Metodología de la Investigación como campo disciplinar y se analiza cómo se produce y reproduce la dicotomía entre lo cualitativo y lo cuantitativo en Sociología. Este equipo hizo un análisis a partir de la producción sobre metodología presentada en congresos y publicada en revistas científicas, así como un análisis bibliográfico de los programas de distintas cátedras de metodología, identificando los autores más utilizados, su origen nacional y la antigüedad de los textos. Otros trabajos posteriores continuaron la línea de investigación analizando planes de estudios, espacios curriculares y programas (Lacchini, Quiroga-Branda, y Vestfrid, 2012; Piovani, Rausky, y Santos, 2012; Vestfrid y Torillo, 2012).

\footnotetext{
${ }^{2}$ Este artículo constituye un resultado de la indagación en el marco proyecto de Reconocimiento Institucional (Facultad de Ciencias Sociales - Universidad de Buenos Aires) «Evaluando recursos pedagógicos para la articulación de teoría y metodología de la investigación». Algunas de las reflexiones aquí vertidas fueron presentadas y discutidas en las Jornadas de Sociología de la UBA de 2019.
} 
En América Latina, los antecedentes en la temática de enseñanza de metodología han versado sobre ejes como las prácticas docentes en el aula, la mirada de los estudiantes y los perfiles profesionales que la formación produce (De-Sena, 2013; Moreno y Morales, 2014; Seid, 2012). Asimismo, ha habido aportes que situaron estas inquietudes en el horizonte de las especificidades históricas de las ciencias sociales latinoamericanas (Gallegos-Elías, Mejía-Martínez, y Paredes-Vilchiz, 2016; Piovani, 2011).

En países anglosajones se ha señalado algo que también parece aplicar en Latinoamérica: la investigación sobre la enseñanza-aprendizaje de metodología de la investigación a menudo se limita a reflexiones sobre las propias prácticas de los docentes, y a evaluaciones o estudios de caso de determinadas cohortes, contextos o desafíos (Earley, 2013). Se ha afirmado que las concepciones de los estudiantes sobre la naturaleza del conocimiento impactan en el estudio y en el proceso de aprendizaje de la investigación social. Las distintas orientaciones metodológicas, bien fundamentadas por separado en diferentes abordajes epistemológicos, por lo general no han llegado a ser integradas por la comunidad de investigadores de modo que permitan que los estudiantes construyan una idea coherente de la metodología de la investigación (Obwuegbuzie y Leech, 2003).

Las visiones mistificadas de la actividad científica pueden ser reproducidas en algunos manuales de metodología que ofrecen descripciones abstractas de la investigación, a modo de recetas. Asimismo, cuando se da por sentado que los docentes de metodología saben qué es la investigación y cómo enseñarla, se dificulta que los estudiantes comprendan la existencia de diversas concepciones o maneras de entender qué es la investigación (Murtonen y Lehtinen, 2005). En Australia, se han identificado al menos cuatro concepciones distintas (Brew, 2001): una concepción "dominó", en la que técnicas y actividades separadas se sintetizan para resolver un problema; una concepción del "velo", en la que lo fundamental es el descubrimiento de procesos y mecanismos ocultos; una concepción "mercantil", en el sentido de un proceso de producción con insumos, productos e intercambios; y una concepción "aventurera" donde se representa la investigación como un viaje en el cual quien investiga conoce a la vez el mundo y a sí mismo.

\section{METOdOLOGÍA}

Tomando en consideración que existen distintos caminos para indagar acerca de la enseñanza de la investigación en Sociología, nuestro interés fue analizar programas de materias relacionadas con la metodología de la investigación social. Ante la imposibilidad de realizar observaciones en el espacio áulico en numerosas universidades distantes geográficamente, consideramos que -como modo de aproximación a la temática- el análisis de programas nos da pistas (o indicios) acerca de los procesos de enseñanza/aprendizaje de metodología. Aunque con fines expositivos mencionaremos las universidades a las que pertenecen los programas analizados, destacamos que no necesariamente son representativos de las universidades, ni tampoco de las cátedras puesto que los programas son modificados periódicamente-. Los programas de materias integran los saberes legitimados con proyectos teóricos e intenciones pedagógicas. Nos interesan porque constituyen una forma posible, entre otras, de hacer observables prácticas y discursos involucrados en la formación en investigación.

En tal sentido, los programas de distintas materias metodológicas del año 2018 (o el más próximo que se halló) fueron las unidades de análisis de la investigación que hemos desarrollado, cuyos principales resultados damos a conocer en este artículo. Más concretamente, cada uno de los programas fue trabajado como documento, enfatizando aquello que es enunciado discursivamente y teniendo en cuenta que, en su carácter de dispositivo didáctico-pedagógico, son habilitantes de unas prácticas en detrimento de otras. 
Fueron analizados un total de sesenta programas entre dieciséis universidades. Se incluyó al menos un programa de cada una de las doce universidades públicas de Argentina donde se dicta la carrera de Sociología. La idea original era centrarse exclusivamente en Argentina. Sin embargo, en el devenir de la indagación, se consideró que los programas de las materias argentinas se comprenderían mejor si se añadiesen al corpus algunos programas de otros países a modo de "control", para tener consciencia de posibles especificidades nacionales. La disponibilidad de este tipo de documentos en la web brinda la oportunidad de ampliar la muestra indefinidamente, pero también obliga a decidir algún recorte en aras de la factibilidad. Las universidades fuera de Argentina fueron seleccionadas por criterios de calidad académica, por encontrarse bien posicionadas en distintos rankings de universidades en las carreras de Sociología.

Del primer conjunto, se relevaron programas de la Universidad de Buenos Aires, la Universidad Nacional del Comahue, la Universidad Nacional de Córdoba, la Universidad Nacional de Cuyo, la Universidad Nacional de La Plata, la Universidad Nacional del Litoral, la Universidad Nacional de Mar del Plata, la Universidad Nacional de San Juan, la Universidad Nacional de San Martín, la Universidad Nacional de Santiago del Estero, la Universidad Nacional de Tierra del Fuego, y la Universidad Nacional de Villa María. De otros países, por su parte, seleccionamos la Universidad Nacional Autónoma de México, la Universidad Complutense de Madrid, la Universitat de Barcelona y la Università di Bologna.

Para comenzar el análisis, los documentos fueron grillados según el lugar que ocupa la materia dentro del plan de estudios, sus contenidos y la concepción de investigación que subyace en los programas - no fueron el principal objeto de análisis, pero sí aspectos necesarios para contextualizar-. La dimensión central de análisis fueron las propuestas de enseñanza enunciadas en los programas, especialmente las prácticas de investigación como modalidad de ejercicio y de evaluación, y - subsidiariamente- el peso atribuido a las herramientas informáticas en el dictado de la materia

A partir de estos ejes, se efectuaron procedimientos de codificación -abierta, axial y selectiva- de datos textuales, siguiendo el método de la Teoría Fundamentada en su versión de Strauss y Corbin (2002). La categoría central a la que se arribó en la fase de síntesis se denominó, en lenguaje coloquial dentro del equipo de investigación, "la práctica como comodín", en alusión a la ubicuidad y polisemia del término en los programas analizados.

\section{RESULTADOS}

A continuación, se presentan los principales resultados del análisis, organizados en dos ejes. En la primera parte se describe el panorama general de propuestas de enseñanza y modalidades de trabajo encontradas en los programas de materias metodológicas analizados. Luego, se discute acerca de las tensiones que atraviesan a los programas de materias metodológicas, especialmente respecto al lugar de lo práctico de la investigación en la enseñanza.

\subsection{Las variantes en la enseñanza de investigación}

Las materias metodológicas son varias - más de una- en todos los planes de estudio de las carreras de Sociología examinadas. Por ende, se cursan a lo largo de varios semestres. En la mayoría de los casos la primera Metodología de la Investigación se ubica en el segundo año de las carreras. En algunos casos, en primer año hay una materia que también remite directa o indirectamente al dominio de lo metodológico, como Metodología de las Ciencias Sociales, cuyos contenidos son generalmente epistemológicos. En este sentido, los contenidos epistemológicos, así como también los de estadística, en ocasiones aparecen incluidos en las materias estrictamente metodológicas y en otras constituyen materias aparte. Típicamente, los planes de estudio prevén 
que en segundo año empiecen a estudiarse cuestiones de metodología general, para pasar luego a técnicas más específicas y, en el último año de la carrera, a talleres de investigación.

Los contenidos de los programas analizados versan sobre la metodología de la investigación tal como habitualmente se la entiende en Sociología. Esto implica una preponderancia o exclusividad de la investigación empírica - es decir, están casi ausentes "métodos filosóficos" de investigación, como el dialéctico, el hermenéutico o el fenomenológico- y, dentro de la investigación empírica, una primacía de la investigación de campo - es decir, suelen quedar relegadas la investigación documental y la experimental-. Los núcleos de contenidos más típicos son: formulación de problema, hipótesis y objetivos, selección de casos, técnicas de relevamiento, y formas de análisis. En general estos aspectos se presentan separadamente para el enfoque cuantitativo y para el cualitativo, por lo común en distintas materias, pero a veces dentro de una misma. Si se toman en conjunto las materias metodológicas de una carrera, hay un claro predominio de las técnicas de relevamiento en las unidades de los programas, lo que hace presumir que son los contenidos a los que más tiempo se les dedica.

La manera de entender la investigación que expresan los programas a menudo no es explícita, pero puede inferirse. El protagonismo de las técnicas entre los objetivos y contenidos de la materia permite suponer que se concibe a la investigación como una actividad altamente técnica. Por ejemplo:

- Adquisición por parte del alumnado de los conocimientos necesarios para diseñar una investigación sociológica, eligiendo y combinando, los métodos y técnicas más adecuados para cada situación.

- Adquisición por parte del alumnado de los conocimientos y capacidades necesarios para organizar y ejecutar (gestionar) las distintas fases de un proyecto de investigación social (...).

Objetivos de la materia Introducción a los procesos de la investigación social, Grado en Sociología. Universidad Complutense de Madrid

(resaltado nuestro).

Sin embargo, hay programas, incluso de materias que se ocupan fundamentalmente de metodología cuantitativa, que ponen el acento en los desafíos de articular teoría y metodología, o en la relación entre los aspectos técnicos y las disposiciones subjetivas del investigador.

- que los estudiantes se ejerciten en la construcción del objeto de estudio y el diseño de una investigación empírica;

- que los estudiantes puedan establecer relaciones entre la práctica de la investigación y diferentes niveles de reflexiones teóricas, epistemológicas y metodológicas.

Objetivos de la materia Metodología de la Investigación Social I, Licenciatura en Sociología. Universidad Nacional de La Plata (resaltado nuestro).

Las modalidades de enseñanza enunciadas en los programas pueden tipificarse entre dos polos: conceptual-expositiva y teórico-práctica. En el primero, los programas prevén que el docente exponga los contenidos de la materia, es decir, que desarrolle verbalmente la lógica general y los principales métodos de investigación, con énfasis en las lecturas obligatorias. Se infiere que las materias que proponen la modalidad conceptual lo hacen porque luego los estudiantes cursan talleres destinados a la puesta en práctica de las habilidades para investigar. En el otro polo, se observan programas 
-la mayoría- que proponen una combinación de clases expositivas y clases prácticas. Estas propuestas pedagógicas orientadas a la práctica se basan, como afirma uno de los programas de Metodología de la Investigación Social de la Universidad Nacional de Córdoba (Argentina), en la constatación de que "el mero cursado de asignaturas metodológicas no es suficiente para lograr esta apropiación; considerando que la investigación es una práctica, su dominio sólo puede lograrse por medio de la aplicación de procedimientos en contextos concretos".

Una primera modalidad de práctica son los ejercicios puntuales, en general para aprender procedimientos. Una modalidad de ejercitación habitual es el análisis de informes de investigación, que permiten la familiarización con los distintos componentes del proceso la investigación, al menos aquellos plasmados en los resultados escritos. Otros ejercicios típicos versan sobre aspectos específicos de la elaboración de un proyecto, como la formulación de objetivos e hipótesis. Los ejercicios más recurrentes son los vinculados a la preparación y ejecución de experiencias de campo -pequeña encuesta, entrevista, observación-. Para enseñar la fase de análisis cuantitativo, típicamente se propone la resolución de problemas estadísticos que se presentan en una guía de ejercicios. Asimismo, en algunos casos se proponen ejercicios para el dominio básico de programas informáticos que asisten el análisis de datos cuantitativos y cualitativos.

En un segundo nivel de práctica, pueden situarse propuestas de actividades como "experiencia de campo", o "simulación de diseño de investigación". Por ejemplo, en el programa de Taller de métodos y técnicas de investigación social de la Universidad Nacional de Villa María (Argentina) se señala, como uno de los procesos concurrentes del proceso de enseñanza-aprendizaje, la realización de: "un ensayo de la experiencia de investigación". En Metodología de la Investigación I de la Universidad Nacional del Litoral (Argentina), si bien se explicita que el "modelo pedagógico tradicional utilizado para enseñar metodología de la investigación es que se integre al proceso concreto de investigación", las actividades son trabajos prácticos - presumiblemente acotados, por ejemplo, sobre operacionalización y elaboración de instrumentos de registro. También en materias con propósitos "técnicos" hay estrategias didácticas similares: en el programa de un taller de la Universidad Nacional de La Plata (Argentina) titulado Herramientas informáticas para el análisis en ciencias sociales: aplicaciones de técnicas cuantitativas-, se destaca: "La mejor manera de aprender los contenidos y las posibilidades de las técnicas metodológicas no es explicarlas de manera abstracta sino aplicarlas en situaciones concretas de la práctica de investigación sociológica”. Este tipo de prácticas pueden situarse en un punto intermedio entre los ejercicios puntuales o aislados y la realización de una investigación completa.

En varias materias metodológicas en Argentina (universidades de Buenos Aires, La Plata, Cuyo, Tierra del Fuego) se propone un tipo de práctica de alcance mayor: un pre-proyecto o ante-proyecto de investigación, en el marco del cual se aprenden contenidos muy diversos. La justificación pedagógica remite a la necesidad de que las decisiones teóricas, metodológicas y técnicas se perciban articuladamente. Asimismo, el aprendizaje enmarcado en un proyecto permitiría la comprensión del proceso de investigación en su conjunto, al hacerse evidente la relación entre etapas. Los alumnos deben formular preguntas, objetivos e hipótesis a la vez que construyen un marco teórico. A continuación, determinar estrategias metodológicas y vincularlas con la teoría social. Luego los alumnos deben realizar trabajo de campo, por ejemplo, construir un cuestionario, aplicarlo y procesarlo. Por último, analizar e interpretar la información en función de la problemática teórica y redactar un informe. Mediante sucesivas presentaciones grupales los estudiantes deben exponer los avances de cada fase. En este recorrido se busca que los estudiantes se enfrenten, con la guía del docente, a los desafíos que supone investigar. En cierto sentido, la enseñanza mediante proyectos de investigación constituye la modalidad que pone en acto de manera más acabada el 
apotegma "aprender a investigar investigando".

A partir de los anteproyectos de investigación se promueve pedagógicamente la reflexividad en la producción de conocimiento. Estas propuestas se basan en una pedagogía de la investigación "con dimensiones multi e interdimensionales, que contiene una parte teórica y reflexiva, una parte técnica, una parte descriptiva, una parte creativa e innovadora y una parte normativa" (Trejo-García y García-Córdoba, 2009, 137) mediante la cual la aproximación al modus operandi científico se aprendería en el hacer. Una particularidad de las materias que exigen la realización de un anteproyecto de investigación por parte de los estudiantes, sobre todo cuando esa práctica cuenta con una tutorización, reside en que no es sencillo estipular de antemano cuánto trabajo se pedirá, cuán lejos o qué tan seriamente se tomará la actividad. En algunos programas se resalta que se trata de prácticas "reales" de investigación, tal vez anticipándose a la suspicacia de que las prácticas en las materias metodológicas constituyan un "como si” de la investigación.

Algunas cátedras que desarrollan la estrategia pedagógica de llevar a cabo un anteproyecto de investigación poseen una modalidad de cohortes, es decir, una estructura de varias materias metodológicas que se plantean en conjunto y en un desarrollo sucesivo. La continuidad de las materias, típicamente tres, plantea la realización de una misma investigación en etapas, comenzando en la primera materia de metodología para concluirlo en la última. Allí se busca enseñar en cada una de ellas distintas etapas de la investigación, o bien las metodologías cuantitativas, las cualitativas y su articulación (Universidad de Buenos Aires). Así, se busca abordar una misma problemática de investigación según distintos objetivos y estrategias metodológicas.

Desplazándonos al continente europeo, a partir de los documentos analizados de la Universidad Complutense de Madrid podemos destacar - para la materia Métodos y técnicas de investigación social cuantitativas- que se prevé la ejercitación de todas las decisiones de un estudio por encuesta. El programa de Métodos y técnicas de investigación social cualitativos, por su parte, prevé que los estudiantes ejercitarán cada una de las principales técnicas. En Barcelona, Tècniques d'Investigació Social III y IV ofrecen contenidos de análisis estadístico avanzado, con ejercicios de procesamiento de información en ordenador. En otros programas analizados también se subraya la centralidad de la práctica, pero no encontramos detalles. De manera ilustrativa, en uno de los documentos de la Università di Bologna se destaca que el "objetivo es que los estudiantes realicen una actividad de investigación", pero no se hace aclaración sobre el tema ni los distintos pasos en que se desagregaría la actividad.

El caso de la Universidad Nacional Autónoma de México resultó revelador, ya que los contenidos metodológicos están distribuidos en más de diez materias, algunas de las cuales son muy específicas, por ejemplo, Procesamiento de datos. Por lo tanto, al igual que en las carreras europeas analizadas, predominan las ejercitaciones separadas para cada contenido. Cuando los contenidos metodológicos en una carrera y al interior de una materia están más fraccionados, se tiende a la cobertura de más contenidos, pero se diluye la centralidad de la construcción del objeto y de la teoría social, ya que el acento termina poniéndose en el entrenamiento para el dominio de cada técnica. Esto contrasta con las modalidades mediante anteproyectos, donde se privilegia el sentido de las metodologías y técnicas en relación con problemáticas teóricas.

En contrapartida, los anteproyectos omnímodos presentan una desventaja decisiva: excluyen varias técnicas de la puesta en práctica. Por ejemplo, en ninguna cátedra de Metodología de la Investigación Social de la Universidad de Buenos Aires, donde se trabaja con esta modalidad, se prevé la realización de un grupo de discusión ni la aplicación de técnicas "avanzadas" de análisis estadístico - y en una cátedra incluso está ausente la investigación documental.

Respecto al peso atribuido a las herramientas informáticas, el panorama es diverso. Para el año 2018, en pocos programas argentinos, excepto los de materias específicas de 
informática, se especificaba el papel de estas herramientas y la manera concreta en que se preveía integrarlas en la enseñanza. Por ejemplo, en el programa de Metodología de las Ciencias Sociales (Universidad Nacional de Cuyo, Argentina), se señala que el dictado de la materia se llevará a cabo a través de una estrategia pedagógico-didáctica que privilegia la enseñanza mediante el uso de dispositivos informáticos, pero no hay mayores especificaciones. Lo que predomina en los programas argentinos es la mención de herramientas informáticas para ejercicios puntuales, muchas veces a modo de complemento. En las carreras europeas, en cambio, hay más materias diseñadas para la aplicación de técnicas en un laboratorio de informática. Por ejemplo, en Tècniques d'Investigació Social III (Universitat de Barcelona) a las clases prácticas se las denomina "de ordenador con tutoría dirigida". Incluso la bibliografía revela el peso atribuido a tales herramientas, pues se incluyen distintos manuales de usuario de programas informáticos para la investigación.

\subsection{El consenso sobre la importancia de la práctica y las divergencias en las prácticas}

Difícilmente se encuentren propuestas de enseñanza de metodología que no reconozcan la necesidad de la práctica para adquirir el saber hacer de la investigación. Cuando se omite o no se enfatiza en este aspecto, en general se trata de programas de materias de carácter general, como Metodología de las Ciencias Sociales, cuyos contenidos son ante todo epistemológicos. Ahora bien, los programas de materias específicas sobre investigación, que siempre subrayan la importancia de la instancia práctica, pueden concebirla de distinto modo. Una práctica puede ser, por ejemplo, el análisis de investigaciones ya realizadas y la discusión de informes de investigación. En las metodologías cuantitativas o en materias de estadística aplicada a la investigación, las prácticas típicamente consisten en la resolución de guías de ejercicios. En algunas materias de metodología cualitativa la práctica consiste en realizar experiencias puntuales de trabajo de campo. En otros casos se asocia la práctica con el uso de herramientas informáticas. Finalmente, en no pocas materias de metodología general se proponen prácticas relativas a elaborar un proyecto de investigación y, dentro de éstas, algunas también proponen llevarlo a cabo.

Cabe advertir la confusión que el lenguaje usual en los programas de enseñanza parece inducir: no es lo mismo hablar de la práctica como modalidad de trabajo para el proceso de enseñanza-aprendizaje que hablar de una práctica de investigación. En este sentido, por ejemplo, los ejercicios de reconocimiento de componentes y etapas de investigación a partir de lectura y análisis metodológico de informes o artículos pueden ser prácticas solamente desde el punto de vista pedagógico. En el caso de los ejercicios de reconocimiento y aplicación de técnicas o procedimientos, lo práctico desde el punto de vista de modalidad de la enseñanza puede considerarse también una incipiente práctica investigativa. Por ejemplo, en algunas materias de estadística para la investigación se proponen prácticas de análisis estadístico de datos secundarios en el gabinete de computación, empleando bases usuarias de institutos de estadística o matrices de datos provenientes de investigaciones realizadas por los docentes.

La aplicación de los procedimientos puede servir para ilustrar lo desarrollado en clases expositivas, para replicar resultados previstos -a la manera de simulación de una investigación- o para imponer un dispositivo que pueda recorrerse de distintas maneras, dejando cierto margen de libertad y creatividad. Las experiencias puntuales de trabajo de campo, especialmente de tipo cualitativo, ya parecen ser algo más que aplicación. Sin embargo, que constituya una práctica de investigación propiamente dicha dependerá del grado de reflexividad que se promueva y se alcance. Un interesante ejemplo de este tipo de práctica se realiza en una materia de metodologías cualitativas en la Universidad Nacional de La Plata, donde la actividad que se plantea como "experiencia de trabajo de campo", abarca diseño, registro y análisis de información, es 
decir, condensa de alguna manera los momentos básicos del proceso de investigación.

En lo que respecta a las prácticas que recurren a un proyecto de investigación, en algunas materias metodológicas la formulación del proyecto se lleva a cabo a lo largo de todo el curso, en un proceso de sucesivas reelaboraciones hasta la entrega final. Como evidente ventaja de esta propuesta, se destaca el hecho de darle a la construcción del objeto de estudio y al diseño de investigación todo el tiempo y la atención posibles. En algunos casos, como en la Universidad Nacional del Comahue (Argentina), se trabaja con esta modalidad, asumiendo que las etapas de ejecución del proyecto se podrán llevar a cabo posteriormente, al momento de realizar la tesis de grado. En cambio, cuando no se trata solamente de formular un proyecto, sino de realizar una práctica que abarque el proceso completo de investigación, se añaden complejidades para la organización de la enseñanza. En general, en estos últimos casos prevalecen las propuestas de trabajos grupales. Algunos equipos docentes proporcionan a los estudiantes un proyecto marco dentro del cual se trabajará, otros consensúan con los estudiantes el tema de investigación y elaboran un proyecto entre todos, otros solicitan a los estudiantes que formulen en grupo sus propios proyectos dentro de un determinado tema y luego "unifican" los distintos proyectos para poder realizar el trabajo de campo en común -imprescindible para las técnicas estandarizadas-.

En sus distintas variantes, las prácticas enmarcadas en un proyecto de investigación ofrecen ventajas relevantes: permiten que los ejercicios para dominar distintas técnicas y procedimientos se vinculen entre sí y no queden descontextualizados, muestran que las decisiones de investigación son decisiones reales que deben ser tomadas y que tienen consecuencias, y habilitan el escenario más propicio para la reflexividad, para la articulación entre epistemología, teoría social, metodología y técnicas. El trabajo en el aula a partir de un proyecto obliga a revisar permanentemente la coherencia entre interrogantes, objetivos, marco conceptual y posibilidades para la producción y análisis de evidencia empírica. En este sentido, el recurso a un proyecto promovería la toma de conciencia a partir de la práctica de que las operaciones de investigación, en especial las técnicas e instrumentos, no pueden aplicarse mecánicamente sin control de supuestos teóricos y epistemológicos. A cambio, este recurso para la enseñanza de metodología insume mayor tiempo y exige poner en juego una variedad de habilidades simultáneas -cuando aún no se las domina y precisamente deberían ser adquiridas mediante la práctica en el proyecto.

Posiblemente el proyecto funcione de distinto modo y tenga distinta gravitación según el tema metodológico que se enseñe. Para el aprendizaje de técnicas específicas, trabajar bajo un proyecto puede hacer más lento y denso el proceso de aprendizaje, en comparación a los ejercicios puntuales que permiten focalizar en cada técnica en particular, de a una por vez, haciendo abstracción momentáneamente de las complejidades que surgen cuando ella se inserta en una investigación concreta. En cualquier caso, parece conveniente recordar que no se puede enseñar todo y de todas las formas posibles. Si se recurre a un proyecto, lo que se gana en profundidad y reflexividad tal vez se pierda en variedad de métodos y técnicas: por más que muchas veces los docentes intenten adecuar los objetivos del proyecto para que, por ejemplo, requieran abordajes cuantitativos y cualitativos, ningún buen proyecto puede tener tanta versatilidad para que todas las técnicas cuadren en él. Al contrario, si se recurre a ejercicios más "descontextualizados", se gana en amplitud de técnicas y procedimientos que pueden llegar a reconocerse, pero se diluyen tanto la práctica real de investigación como el sentido teórico de las técnicas.

\section{CONCLUSIONES}

La enseñanza de metodología supone una modelización de las prácticas reales de investigación. Los manuales y textos de metodología son una especie de "teoría" sobre 
el proceso de investigación: contienen generalizaciones, tipificaciones, clasificaciones $\mathrm{y}$, a menudo, las enuncian en forma de prescripciones. El desafío de las materias es revitalizar esos saberes objetivados -y a veces fosilizados-, retornando de lo conceptual de la metodología a la práctica de la investigación.

Las metodologías y técnicas que se enseñan, así como el tiempo y la importancia que se les asigna, se convierten en modelos de posibilidades de investigación en Sociología. La selección que requiere todo programa de estudio implica decisiones sobre qué se incluye y qué se excluye. Dentro de lo que se decide enseñar explícitamente, el tiempo que se le dedica a cada tema es una de las principales decisiones "políticas" de la enseñanza, así como lo son los propósitos y las modalidades de trabajo, en particular las evaluaciones.

De manera análoga a la enseñanza de los autores clásicos, que la tradición signa como imprescindibles para todo aquel que se inicia en una disciplina de Ciencias Sociales, algunos tipos y técnicas de investigación se convierten en modelos. El trabajo de campo etnográfico en Antropología, la investigación de archivo en Historia, o las técnicas econométricas en Economía, son arquetipos de investigaciones que se consideran "propias" de una disciplina. En el caso de la Sociología, la técnica de encuesta y la entrevista semi-estructurada parecen ser las técnicas más empleadas y más enseñadas, que sobresalen en la escena plural de las metodologías. La formación metodológica en Sociología privilegia la investigación de campo en detrimento de la investigación de biblioteca. La investigación bibliográfica del erudito como momento necesario de toda investigación se relega cuando se da por sentado que se debe armar un estado de la cuestión, sin advertir el hecho de que es una investigación en sí misma, subsidiaria de una investigación mayor -en esto probablemente sean ostensibles las diferencias con otras disciplinas, en particular de la tradición de las Humanidades, como Historia o Letras-.

Dentro de la investigación de campo, algo recurrente en los casos analizados es la división entre los métodos cuantitativos y los cualitativos. Si bien es comprensible que se decida enseñarlos por separado, cabe señalar lo extendido de esta convención en los planes de estudio - que prevén distintas materias para unos y otros-, así como en los bloques de contenidos de una misma materia. En los debates metodológicos esta separación ha sido problematizada hace tiempo (Cohen y Gómez-Rojas, 2019; Cook y Reichardt, 1986), y también se ha planteado que es inadecuada incluso para la enseñanza (Obwuegbuzie y Leech, 2003).

Más allá de los denominadores comunes en los programas analizados -que atribuimos principalmente al hecho de que estén insertos en carreras de Sociología-, destacamos tres dimensiones en las que se encontraron divergencias, que pueden ser entendidas como tensiones inherentes a la enseñanza de la investigación en carreras de grado.

- La tensión entre la necesidad de pluralismo y la de profundización, es decir, entre presentar la multiplicidad de posibilidades y, a la vez, enseñar al menos algunas de esas posibilidades de un modo más exhaustivo, tanto conceptual como práctico.

- La tensión entre la exposición conceptual y la modalidad taller. Hay un amplio consenso en torno a que se aprende a investigar investigando, aunque también es cierto que se aprende metodología de manera conceptual. El equilibrio puede no ser sencillo: si se pasa "demasiado pronto" a las prácticas, puede que se eche en falta la propedéutica que suponen las clases conceptuales sobre metodología. Pero en cuanto estas últimas se prolongan demasiado y, en vez de hacer algo, se pasa a relatar exhaustivamente cómo se hace -por ejemplo, piénsese en la exposición sobre recomendaciones generales para la redacción de preguntas de cuestionario, puede tenerse la sensación de que se está ante las Instrucciones para subir una escalera, de Julio Cortázar. 
- La tensión entre las prácticas para el dominio técnico y las prácticas para la artesanía intelectual. Ambos polos son necesarios, pero las proporciones pueden variar mucho. Esta tensión contiene las divergencias en la manera de entender qué es la investigación y para qué (Brew, 2001), así como el posicionamiento entre los ejercicios puntuales y los anteproyectos omnímodos. También pone de manifiesto la contradicción entre enseñar metodología cumpliendo un cronograma y enseñar a investigar.

Por último, si bien las divergencias encontradas en los programas y las tensiones planteadas pueden exponer disposiciones y posicionamientos respecto a la enseñanza de investigación, creemos que todas las estrategias tienen desventajas y ninguna es inherentemente superior. Desde nuestra perspectiva, la situación ideal es que los estudiantes experimenten también la diversidad de modalidades de enseñanza a lo largo de sus carreras.

\section{A. APÉNDICE}

Universidad Nacional Autónoma de México - Licenciatura en Sociología

- Metodología I. La metodología en los Clásicos

- Metodología II. La metodología contemporánea

- Metodología

- Estadística descriptiva

- Estadística inferencial

- Análisis cuantitativo

- Procesamiento de datos

- Taller de Investigación Sociológica I

Universidad Complutense de Madrid - Grado de Sociología

- Filosofía de las Ciencias Sociales

- Introducción a los procesos de la investigación social

- Estadística descriptiva aplicada a las Ciencias Sociales

- Métodos y técnicas de investigación social cuantitativas

- Métodos y técnicas de investigación social cualitativos

Universitat de Barcelona - Grado de Sociología

- Metodologia de les Ciències Socials

- Tècniques d'Investigació Social I

- Tècniques d'Investigació Social II

- Tècniques d'Investigació Social III

- Tècniques d'Investigació Social IV

Università di Bologna - Laurea in Sociologia

- Metodologia e tecniche della ricerca sociale 
- Laboratorio di ricerca sociale qualitativa 1

- Laboratorio di ricerca sociale quantitativa 1

- Laboratorio di ricerca sociale qualitativa 2 e di intervento sociale

- Laboratorio di ricerca sociale qualitativa 2

EN ARGENTINA:

Universidad de Buenos Aires - Licenciatura en Sociología

- Metodología de la Investigación Social I (cátedra Infesta)

- Metodología de la Investigación Social I (cátedra Moreno)

- Metodología de la Investigación Social I (cátedra Sautu)

- Metodología de la Investigación Social II (Infesta)

- Metodología de la Investigación Social II (Moreno)

- Metodología de la Investigación Social II (Sautu)

- Metodología de la Investigación Social III (Infesta)

- Metodología de la Investigación Social III (Moreno)

- Metodología de la Investigación Social III (Sautu)

- Muestreo aplicado a las Ciencias Sociales

Universidad Nacional del Comahue - Licenciatura en Sociología

- Metodología II

Universidad Nacional de Córdoba - Licenciatura en Sociología

- Metodología de la Investigación Social I - Cuantitativa

Universidad Nacional de Cuyo - Licenciatura en Sociología

- Metodología de las Ciencias Sociales

- Técnicas Estadísticas para la Investigación Social

- Técnicas cualitativas de Investigación Social

Universidad Nacional de La Plata - Licenciatura en Sociología

- Metodología de la Investigación Social I

- Metodología de la Investigación Social II. Métodos y técnicas cualitativas

- Socioestadística

- Herramientas informáticas para el análisis de datos cualitativos en ciencias sociales con ATLAS.ti

- Herramientas informáticas para el análisis en ciencias sociales: aplicaciones de técnicas cuantitativas

Universidad Nacional del Litoral - Licenciatura en Sociología

- Métodos Estadísticos para Ciencias Sociales 
- Metodología de la Investigación I

- Metodología de la Investigación II

Universidad Nacional de Mar del Plata - Licenciatura en Sociología

- Metodología Cuantitativa I

- Metodología Cualitativa II

Universidad Nacional de San Juan - Licenciatura en Sociología

- Metodología de la Investigación I

Universidad Nacional de San Martín - Licenciatura en Sociología

1. Metodología de la Investigación

Universidad Nacional de Santiago del Estero - Licenciatura en Sociología

- Estadística I

- Metodología de la Investigación I

- Metodología de Investigación II

Universidad Nacional de Tierra del Fuego - Licenciatura en Sociología

- Metodología de Investigación en Ciencias Sociales

- Metodología de la Investigación I

- Taller de Investigación II

- Estadística

Universidad Nacional de Villa María - Licenciatura en Sociología

- Taller de Investigación

- Taller de métodos y técnicas de investigación social

- Estadística aplicada a las ciencias sociales

\section{REFERENCIAS}

Becker, H. S. (2009). Trucos del oficio: cómo conducir su investigación en ciencias sociales. Buenos Aires: Siglo Veintiuno.

Bourdieu, P., Chamboredon, J. C., y Passeron, J. C. (2008). El Oficio de Sociólogo. Presupuestos epistemológicos. Buenos Aires: Siglo Veintiuno.

Bourdieu, P., y Gros, F. (1998). Principios para una reflexión sobre los contenidos de la enseñanza. En P. Bourdieu (Ed.), Capital cultural, escuela y espacio social. Madrid: Siglo XXI.

Bourdieu, P., y Passeron, J. C. (2004). Los herederos. Los estudiantes y la cultura. Buenos Aires: Siglo Veintiuno.

Brew, A. (2001). Conceptions of Research: A phenomenographic study. Studies in Higher Education, 26(3), 271-285. https://dx.doi.org/10.1080/03075070120076255

Cohen, N., y Gómez-Rojas, G. (2019). Metodología de la investigación, ¿para qué? La producción de los datos y los diseños. Buenos Aires: Teseo-CLACSO. 
Cohen, N., y Piovani, J. I. (Eds.). (2008). La metodología de la investigación en debate. Argentina: Edulp / Eudeba.

Cook, T. D., y Reichardt, C. S. (1986). Métodos cualitativos y cuantitativos en investigación evaluativa. Madrid: Morata.

De-Sena, A. (2013). Estudiantes de sociología: ¿herederos como siempre? En F. Nievas (Ed.), Mosaico de sentidos. Vida cotidiana, conflicto y estructura social (pp. 153-170). Buenos Aires: Estudios Sociológicos. Descargado de http://estudiosociologicos.org/portal/ mosaico-de-sentido/

Earley, M. A. (2013). A synthesis of the literature on research methods education. Teaching in Higher Education, 19(3), 242-253. https://doi.org/10.1080/13562517.2013.860105

Gallegos-Elías, C., Mejía-Martínez, A., y Paredes-Vilchiz, Y. (Eds.). (2016). ¿Cómo investigamos? ¿Cómo enseñamos a investigar? Tomo IV. México: UNAM. https:// doi.org/10.22201/fcpys.9786070279256p.2016

Gómez-Rojas, G., y Malegarie, J. (2007). Cuestiones metodológicas: una mirada de los alumnos. XXVI Congreso de la Asociación Latinoamericana de Sociología, Guadalajara (México), Asociación Latinoamericanade Sociología. Descargado de http://cdsa .aacademica.org/000-066/1145.pdf

Lacchini, A. J., Quiroga-Branda, J., y Vestfrid, P. (2012). Enseñando a investigar. Modas en la trastienda. VIII fornadas de Sociología de la Universidad Nacional de La Plata, La Plata. Descargado de http://jornadassociologia.fahce.unlp.edu.ar/viii-jornadas/viii-jornadas -2014/PON09\%20Lacchini.pdf/view? searchterm=None

Moreno, M., y Morales, N. (2014). La formación metodológica desde la perspectiva de los estudiantes. Consideraciones alrededor de la sociología, la metodología y la proyección laboral. Entramados y perspectivas. Revista de la Carrera de Sociología, 4(4), 115-144. Descargado de https://publicaciones.sociales.uba.ar/index.php/ entramadosyperspectivas/article/view/530/468

Murtonen, M., y Lehtinen, E. (2005). Conceptions of Research and Methodology Learning. Scandinavian fournal of Educational Research, 49(3), 217-224. https://dx.doi.org/10 $.1080 / 00313830500109519$

Obwuegbuzie, A. J., y Leech, N. L. (2003). Taking the 'Q' out of research: Teaching research methodology courses without the divide between quantitative and qualitative paradigms. Quality and Quantity, 39, 267-295. https://doi.org/10.1007/s11135-004-1670 $-0$

Piovani, J. I. (2011). La Metodología de las Ciencias Sociales como campo de interés intelectual en América Latina. Revista latinoamericana de metodología de las ciencias sociales, 1(1), 1-7. Descargado de https://dialnet.unirioja.es/descarga/articulo/3869751 .pdf

Piovani, J. I., Rausky, M. E., y Santos, J. (2012). La enseñanza de la metodología en posgrados universitarios de Ciencias Sociales de Argentina. VII fornadas de Sociología de la Universidad Nacional de La Plata, 5-7 de diciembre, La Plata (Argentina). Descargado de http://sedici.unlp.edu.ar/bitstream/handle/10915/30267/Documento_completo.pdf ?sequence $=1 \&$ is Allowed $=y$

Rancière, J. (2007). El Maestro Ignorante. Cinco lecciones sobre la emancipación intelectual. Buenos Aires: Libros del Zorzal.

Rizo-García, M. (2006). Experiencias de investigación en comunicación con estudiantes de la Licenciatura en Comunicación y Cultura de la Universidad Autónoma de la Ciudad de. En J. A. Dettleff (Ed.), Desafíos de la investigación universitaria (pp. 19-39). Perú: Departamento Académico de Comunicaciones, Pontificia Universidad Católica del Perú.

Seid, G. (2012). Las particularidades de las asignaturas metodológicas desde las perspectivas de los estudiantes. En J. Piovani, M. Carreño, y J. Castellanos (Eds.), Memorias del III Encuentro Latinoamericano de Metodología de las Ciencias Sociales (ELMeCS) (pp. 145161). Manizales: Universidad de Caldas/Universidad de Manizales/RedMet. 
Strauss, A., y Corbin, J. (2002). Bases de la investigación cualitativa: técnicas y procedimientos para desarrollar la teoría fundamentada. Colombia: Editorial Universidad de Antioquia.

Trejo-García, M., y García-Córdoba, F. (2009). Pedagogía de la investigación.

Metodología de la Ciencia. Revista de la Asociación Mexicana de Metodología de la Ciencia y de la Investigación, 1, 135-141. Descargado de http://www.ammci.org .mx/revista/pdf/Seccion\%20metodologia\%20de\%20la\%20ciencia\%20(2a\%20parte)/ PedagogiaTrejoyCordoba.pdf

Vestfrid, P., y Torillo, D. (2012). La formación en investigación en carreras de ciencias sociales de la UNLP: Análisis de experiencias desde la perspectiva docente. VII fornadas de Sociología de la Universidad Nacional de La Plata, 5-7 de diciembre, La Plata (Argentina). Descargado de http://jornadassociologia.fahce.unlp.edu.ar/vii-jornadas-2012/actas/ Vestfrid.pdf/at_download/file

Wainerman, C., y Sautu, R. (Eds.). (2011). La trastienda de la investigación. Buenos Aires: Manantial. 